\title{
CALCULATION OF STRESS INTENSITY FACTOR FOR AN INTERNAL CIRCUMFERENTIAL CRACK IN A ROTATING FUNCTIONALLY GRADED THICK-WALLED HOLLOW CIRCULAR CYLINDER UNDER THERMAL SHOCK
}

\begin{abstract}
In this article, the fracture behavior of functionally graded thick-walled cylinder under thermo-mechanical shock is investigated. For this purpose, classical coupled thermoelastic equations are used in calculations. First, these equations are discretized with extended finite element method (XFEM) in the space domain and then they are solved by the Newmark method in the time domain. The most general form of interaction integral is extracted for axially symmetric circumferential crack in a cylinder under thermal and mechanical loads in functionally graded materials and is used to calculate dynamic stress intensity factors (SIFs). The problem solution has been implemented in MATLAB software.
\end{abstract}

\section{Introduction}

Functionally graded materials (FGMs) have an important role in the design of various modern structures. The unique features provided of FGMs have induced scientists to study them [1]. These materials are used extensively in applications, such as thermal barrier coatings, in which they are exposed to severe stress gradients including thermal and/or mechanical loading. FGMs can be used in the construction of tanks and cylindrical furnace, such as cement kiln. Circumferential cracks are occasionally developed in cylindrical structures during service or production [2].

Numerous researches have been conducted on the behavior of cracked cylinder. Meshii and Watanabe [3] achieved the closed-form SIFs for limited homogenous

\footnotetext{
${ }^{1}$ Shahrood University of Technology, Shahrood, Iran. Emails: rz.elahi@gmail.com, mmrokhi@shahroodut.ac.ir
} 
cylinder with a circumferential crack. They also examined the effects of the cylinder length and crack position on these factors. Seifi [4] studied the stress intensity factors for surface cracks inside the cylinder made of functional materials by employing the weight function method. Eshraghi and Soltani [5] obtained the stress intensity factors for circumferential cracks inside cylinder made of functional materials using a weight function method. Varfolomeyev et al. [6] calculated mode I stress intensity factors and opening internal crack faces using a weight function for cylinder under axisymmetric uniform loading via employing boundary element method. Chen [7] achieved SIFs and displacements for two different length tracks in the limited cylinder under tension stress.

Jones [8] achieved stress intensity factors due to transient thermal load on the cylinder with internal circumferential crack through implementing the weight function. Birinci et al. [9] investigated a cylinder with various internal and external metal cladding and achieved SIFs for cylinder under axial loading. Grebner and Strathmeier [10] studied a cracked pipe with finite length using FEM. Mode II SIFs were calculated for this pipe under tension loading. Tran and Geniaut [11] studied SIFs for circumferential cracked cylinder by employing the XFEM and energy method. Lewis and Wang [12] analyzed cracked cylinder under various linear loads and calculated T-stress by using FEM and weight function. Ghajar and Nabavi [13] presented the closed-solution of thermal stress intensity factors for thick and thin wall cylinders with internal circumferential crack under steady state thermal loads in which they used the weight function method. Nabavi and Kamyab [14] determined the closed-solution of thermal stress intensity factors using weight function in a thick wall cylinder with circumferential crack. Shariati et al. [15] investigated SIFs four cylinders with circumferential crack made of functional material under dynamic and static loading. The Galerkin method was employed to discretize the motion equations. Tehrani and Talebian [16] studied the analysis of cylindrical pressure vessels made of FGMs under mechanical and thermal loads.

Among the studies which have been done on dynamic fracture of functional graded materials (FGMs) so far, few number concern the annular circumferential cracks in the thick-walled cylinders under heat shocks using classic coupled thermoelastic equations numerically, therefore it is investigated in the present article. The rule of mixture is used to simulate the FGM in this study. The equations are discretized and assembled in the form of a matrix using the extended finite element method (XFEM) in the place domain. Newmark method is used in solving extracted matrix equations. In addition, general formulation for the interaction integral is extracted in the FGM cylinder under mechanical and thermal loading in cylindrical coordinates and the dynamic stress intensity factors are obtained by interaction integral. A few examples which verify the accuracy and precision of written code in MATLAB programming environment have been presented. Finally, the effects of changing the FGM properties, crack depth and rotation of FGM cylinder made of $\mathrm{ZrO}_{2} / \mathrm{Ti} 6 \mathrm{Al}-4 \mathrm{~V}$ are investigated. 


\section{General problem formulation}

\subsection{Governing Equations}

In this paper, an axisymmetric problem is considered. The cylinders are formed by rotation a plane around an axis. In axially symmetric problems it is enough to analyze these transverse plates. Generally, in the axially symmetric problems, properties, load and boundary conditions have to be axisymmetric. Basic equations, which govern the coupled thermoelasticity problems, are presented as the equations of motion and the First Law of Thermodynamics (energy equation), as [17]

$$
\begin{gathered}
\sigma_{i j, j}+B_{i}=\rho \ddot{u}_{i} \\
\left(k_{i j} T_{, j}\right)_{, i}+\rho c_{t} \dot{\theta}+T_{0}\left(1+\frac{\theta}{T_{0}}\right) \beta_{i j} \dot{\varepsilon}_{i j}=R
\end{gathered}
$$

In above equation, $k_{i j}$ is thermal conductivity, $c_{t}$ - specific heat, $\theta$ - temperature change, $R$ - rate of heat production. The equations of motion in cylindrical coordinates can be expressed as follows [18]:

$$
\begin{gathered}
\frac{\partial}{\partial r}\left(r \sigma_{r}\right)-\sigma_{\theta}+r \frac{\partial \tau_{r z}}{\partial z}+r f_{r}=\rho r \frac{\partial^{2} u_{r}}{\partial t^{2}} \\
\frac{\partial\left(r \tau_{r z}\right)}{\partial r}+r \frac{\partial \sigma_{z}}{\partial z}+r f_{z}=\rho r \frac{\partial^{2} u_{z}}{\partial t^{2}}
\end{gathered}
$$

Stress equations in terms of displacement are expressed as follows [18]:

$$
\sigma_{i j}=\lambda \delta_{i j} \operatorname{div} u+2 \mu e_{i j}
$$

In this equation:

$$
\begin{gathered}
\operatorname{div} u=\frac{1}{r} \frac{\partial(r u)}{\partial r}+\frac{\partial w}{\partial z} \\
\lambda=\frac{E v}{(1+v)(1-2 v)} \\
\mu=\frac{E}{2(1+v)}
\end{gathered}
$$

In these two equations $\lambda$ and $\mu$ are Lamé constants and $v$ is Poisson's ratio. According to the Fourier heat conduction law, equation (2) can be rewritten in cylindrical coordinates as follows:

$$
\frac{\partial}{\partial r}\left(r q_{r}\right)+r \frac{\partial q_{z}}{\partial z}+r \rho c \dot{\theta}+r T_{0}\left(1+\frac{\theta}{T_{0}}\right) \beta \dot{\varepsilon}_{i i}-r R=0
$$


If the temperature change $\theta$ is small comparing with the initial temperature of $T_{0}$, equation (9) can be simplified as below:

$$
\frac{\partial}{\partial r}\left(r q_{r}\right)+r \frac{\partial q_{z}}{\partial z}+r \rho c T+r T_{0} \beta \dot{\varepsilon}_{i i}-r R=0
$$

\subsection{Space discretization}

One of the methods of crack analysis is using the extended finite element method (XFEM). The base of XFEM is the finite element method, which has been developed for discontinuity such as cracks. This method uses local enrichment. Consider a crack in the finite element model according to Fig. 1. All grid nodes of finite element model are determined as $N_{A}$. Additionally, the nodes of elements around the crack tip (enriched nodes of crack tip) are specified with $N_{C}$, which are selected by the user. The set of nodes of elements, which are cropped by the crack (enriched nodes), is determined by $N_{H}$. It is worth mentioning that although an element for modeling the crack tip is sufficient, the use of multiple elements can achieve more accurate achieved [19].

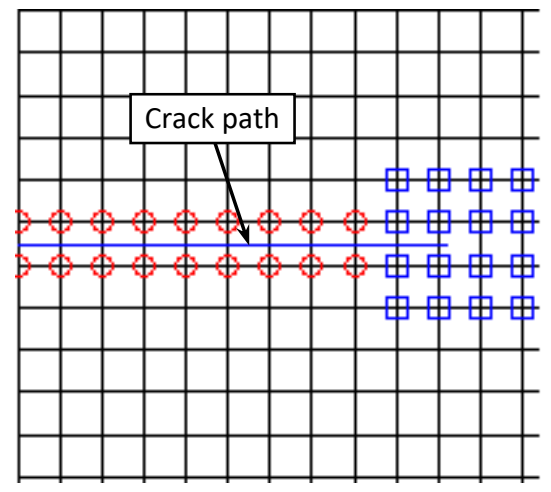

Fig. 1. Selection of enriched nodes for edge crack. Circled nodes are enriched by the discontinuity function whereas the squared nodes are enriched by the crack tip enrichment functions [20]

The displacement field in the XFEM for a fully enriched can be shown as follows [19]:

$$
\begin{aligned}
u(x, y, t)= & \sum_{n \in N_{A}} N_{n}(x, y) a_{n}(t)+\sum_{n \in N_{H}} N_{n}(x, y)\left[H(Z)-H\left(Z_{n}\right)\right] b_{n}(t) \\
& +\sum_{m} \sum_{n \in N_{C}} N_{n}(x, y)\left[F_{m}(r, \varphi)-F_{m}\left(r_{n}, \varphi_{n}\right)\right] c_{n m}(t)
\end{aligned}
$$

In this equation $a_{n}(t), b_{n}(t)$ and $c_{n m}(t)$ will be the nodal unknown vectors which are the functions of time and are defined as follow:

$$
a_{n}(t)=\left\{a_{n}^{u}(t), a_{n}^{v}(t)\right\}^{T}
$$




$$
\begin{gathered}
b_{n}(t)=\left\{b_{n}^{u}(t), b_{n}^{v}(t)\right\}^{T} \\
c_{n m}(t)=\left\{c_{n m}^{u}(t), c_{n m}^{v}(t)\right\}^{T}
\end{gathered}
$$

$H(Z)$ and $F_{m}$ represent Heaviside and crack tip enrichment functions, respectively.

$$
\begin{gathered}
H(Z)= \begin{cases}1, & z>0 \\
0, & z \leqslant 0\end{cases} \\
\left\{F_{m}\right\}=\sqrt{r}\left\{\sin \left(\frac{\varphi}{2}\right), \cos \left(\frac{\varphi}{2}\right), \sin (\varphi) \sin \left(\frac{\varphi}{2}\right), \sin (\varphi) \cos \left(\frac{\varphi}{2}\right)\right\}
\end{gathered}
$$

Finally, the components of the displacement field in XFEM for global $x$ and $y$ coordinates can be written as follows [19]:

$$
\begin{aligned}
u(x, y, t)= & \sum_{n \in N_{A}} N_{n}(x, y) a_{n}^{u}(t)+\sum_{n \in N_{H}} N_{n}(x, y)\left[H(Z)-H\left(Z_{n}\right)\right] b_{n}^{u}(t) \\
& +\sum_{n \in N_{C}} N_{n}(x, y)\left[\sqrt{r} \sin \left(\frac{\varphi}{2}\right)-\sqrt{r_{n}} \sin \left(\frac{\varphi_{n}}{2}\right)\right] c_{n 1}^{u}(t) \\
& +\sum_{n \in N_{C}} N_{n}(x, y)\left[\sqrt{r} \cos \left(\frac{\varphi}{2}\right)-\sqrt{r_{n}} \cos \left(\frac{\varphi_{n}}{2}\right)\right] c_{n 2}^{u}(t) \\
& +\sum_{n \in N_{C}} N_{n}(x, y)\left[\sqrt{r} \sin (\varphi) \sin \left(\frac{\varphi}{2}\right)-\sqrt{r_{n}} \sin \left(\varphi_{n}\right) \sin \left(\frac{\varphi_{n}}{2}\right)\right] c_{n 3}^{u}(t) \\
& +\sum_{n \in N_{C}} N_{n}(x, y)\left[\sqrt{r} \sin (\varphi) \cos \left(\frac{\varphi}{2}\right)-\sqrt{r_{n}} \sin \left(\varphi_{n}\right) \cos \left(\frac{\varphi_{n}}{2}\right)\right] c_{n 4}^{v}(t)
\end{aligned}
$$

If the crack is assumed insulated, the temperature field along the cracks will be discontinuous, and heat flux at the crack tip will be singular. So, to consider the temperature discontinuities, the Heaviside function can be employed. To enrich the crack tip, its temperature fields, which is similar to the displacement field for the crack rupture mode (Mode III), must be checked [19]

$$
T=-\frac{K_{T}}{k} \sqrt{\frac{2 r}{\pi}} \sin \left(\frac{\varphi}{2}\right) .
$$

In this equation, $k$ is the thermal conductivity and $K_{T}$ is the thermal stress intensity factor. Considering the equation above, the temperature field can be discrete, such as displacement field, with the exception that only the first function of the equation (16) is used to enrich the crack tip nodes. So the temperature gradient can be written as follows:

$$
\begin{aligned}
\theta(x, y, t)= & \sum_{n \in N_{A}} N_{n}(x, y) a_{n}^{T}(t)+\sum_{n \in N_{H}} N_{n}(x, y)\left[H(Z)-H\left(Z_{n}\right)\right] b_{n}^{T}(t) \\
& +\sum_{n \in N_{C}} N_{n}(x, y)\left[\sqrt{r} \sin \left(\frac{\varphi}{2}\right)-\sqrt{r_{n}} \sin \left(\frac{\varphi_{n}}{2}\right)\right] c_{n}^{T}(t)
\end{aligned}
$$


$a_{n}^{T}(t), b_{n}^{T}(t)$ and $c_{n}^{T}(t)$ are the amount of temperature change in nods for respective shape function in eq. (19). These equations can be written as [19]:

$$
\begin{aligned}
u(x, y, t)= & \sum_{n \in N_{A}} N_{n}(x, y) a_{n}^{u}(t)+\sum_{n \in N_{H}} \Phi_{n}(x, y) b_{n}^{u}(t) \\
& +\sum_{m=1}^{4} \sum_{n \in N_{C}} \psi_{n m}(x, y) c_{n m}^{u}(t) \\
v(x, y, t)= & \sum_{n \in N_{A}} N_{n}(x, y) a_{n}^{v}(t)+\sum_{n \in N_{H}} \Phi_{n}(x, y) b_{n}^{v}(t) \\
& +\sum_{m=1}^{4} \sum_{n \in N_{C}} \psi_{n m}(x, y) c_{n m}^{v}(t) \\
\theta(x, y, t)= & \sum_{n \in N_{A}} N_{n}(x, y) a_{n}^{T}(t)+\sum_{n \in N_{H}} \Phi_{n}(x, y) b_{n}^{T}(t) \\
& +\sum_{m=1}^{4} \psi_{n 1}(x, y) c_{n 1}^{T}(t)
\end{aligned}
$$

In these equations $\Phi$ and $\psi$ are the enrichment statements of temperature and displacement fields that are responsible for the enrichment of crack path and crack tip, respectively as follows [19]:

$$
\begin{gathered}
\Phi_{n}(x, y)=N_{n}(x, y)\left[H(x, y)-H\left(x_{n}-y_{n}\right)\right] \\
\psi_{n}(x, y)=N_{n}(x, y)\left[\sqrt{r} \sin \left(\frac{\varphi}{2}\right)-\sqrt{r_{n}} \sin \left(\frac{\varphi_{n}}{2}\right), \sqrt{r} \cos \left(\frac{\varphi}{2}\right)-\sqrt{r_{n}} \cos \left(\frac{\varphi_{n}}{2}\right),\right. \\
\sqrt{r} \sin (\varphi) \sin \left(\frac{\varphi}{2}\right)-\sqrt{r_{n}} \sin \left(\varphi_{n}\right) \sin \left(\frac{\varphi_{n}}{2}\right) \\
\left.\sqrt{r} \sin (\varphi) \cos \left(\frac{\varphi}{2}\right)-\sqrt{r_{n}} \sin \left(\varphi_{n}\right) \cos \left(\frac{\varphi_{n}}{2}\right)\right]
\end{gathered}
$$

To solve the equations of motion, the finite element formulation, which is based on Galerkin method can use the. In this method, the space and time functions are divided into distinctive functions. By applying the ratio of residual weighted integrals to weighting function $S_{l}(r, z)$, approximation of Galerkin will be the form of following equations:

$$
\int_{V}\left(\frac{\partial\left(r \sigma_{r}\right)}{\partial r}-\sigma_{\theta}+r \frac{\partial \tau_{r z}}{\partial z}+r f_{r}-r \rho \ddot{u}\right) S_{l} \mathrm{~d} V=0
$$




$$
\int_{V}\left(\frac{\partial\left(r \tau_{r z}\right)}{\partial r}-r \frac{\partial \sigma_{z}}{\partial z}+r f_{z}-r \rho \ddot{w}\right) S_{l} \mathrm{~d} V=0, \quad l=1,2, \ldots, n s
$$

In these equations $S_{l}(r, z)$ is the XFEM shape function. This means that we have:

$$
S_{l}=\left(N_{1}, N_{2}, N_{3}, N_{4}, \varphi_{1}, \varphi_{2}, \varphi_{3}, \varphi_{4}, \psi_{1 m}, \psi_{2 m}, \psi_{3 m}, \psi_{4 m}\right), \quad m=1,2,3,4
$$

Galerkin approximation of the energy equation (10) can be written as follows:

$$
\int_{V(e)}\left(\frac{\partial}{\partial r}\left(r q_{r}\right)+r \frac{\partial q_{z}}{\partial z}+r \rho c \dot{\theta}+r T_{0} \dot{\varepsilon}_{i i}-r R\right) S_{l} \mathrm{~d} V=0
$$

Integral formulation of energy equation (28) for two-dimensional problems can be stated as

$$
\begin{aligned}
& \int_{A(e)}\left(r q_{r} n_{r}\right) S_{l} \mathrm{~d} A-\int_{V(e)} r q_{r} S_{l, r} \mathrm{~d} V+\int_{A(e)}\left(r q_{z} n_{z}\right) S_{l} \mathrm{~d} A \\
& -\int_{V(e)} r q_{z} S_{l, z} \mathrm{~d} V+\int_{V(e)}(r \rho c \dot{\theta}) S_{l} \mathrm{~d} V+\int_{V(e)}\left(r T_{0} \beta \dot{\varepsilon}_{i i}\right) S_{l} \mathrm{~d} V \\
& +\int_{V(e)}(r R) S_{l} \mathrm{~d} V=0, \quad l=1,2, \ldots, n s \\
& \int_{V(e)} r k \theta_{, r} S_{l, r} \mathrm{~d} V+\int_{V(e)} r k \theta_{, z} S_{l, z} \mathrm{~d} V+\int_{V(e)}\left(r \rho c \dot{\theta} S_{l} \mathrm{~d} V\right. \\
& +\int_{V(e)} r T_{0} \beta S\left(\frac{\dot{u}}{r}+\frac{\partial \dot{u}}{\partial r}+\frac{\partial w}{\partial z}\right) S_{l} \mathrm{~d} V=-\int_{A(e)}\left(r q_{r} n_{r}\right) S_{l} \mathrm{~d} A \\
& -\int_{A(e)}\left(r q_{z} n_{z}\right) S_{l} \mathrm{~d} A-\int_{V(e)}(r R) S_{l} \mathrm{~d} V=0, \quad l=1,2, \ldots, n s
\end{aligned}
$$

Equations (19) and (20) can be assembled in the form of a matrix equation. This equation is the coupled XFEM equation, which is obtained as below

$$
[M]\{\ddot{\Delta}\}+[C]\{\dot{\Delta}\}+[K]\{\Delta\}=\{F\}
$$

In this equation $[M],[C]$ and $[K]$ are matrices of mass, damping and stiffness, respectively. $\{\Delta\}$ and $\{F\}$ are nodal unknown vectors and nodal forces vector, respectively. Matrices and vectors used in these equations for a four-node element are presented in appendix. 
Properties matrix in the case of axially symmetric is such as plane strain case that it will show as follows:

$$
[D]=\frac{E}{(1+v)(1-2 v)}\left[\begin{array}{cccc}
1-v & v & v & 0 \\
v & 1-v & v & 0 \\
v & v & 1-v & 0 \\
0 & 0 & 0 & \frac{1-2 v}{2}
\end{array}\right]
$$

\subsection{Time integration}

Using step-by-step numerical methods is very frequent especially in nonlinear dynamical analysis. The most common methods are the direct time methods, so that the equilibrium equations can be solved at discrete times. Choosing the size of time step used for step-by-step calculation of system dynamic response is limited to the stability or accuracy of the algorithm. Newmark method is one of the numerical methods for second order differential equations.

$$
\begin{gathered}
{[M]\left\{\ddot{\Delta}_{n+1}\right\}+[C]\left\{\dot{\Delta}_{n+1}\right\}+[K]\left\{\Delta_{n+1}\right\}=\left\{F_{n+1}\right\}} \\
\left\{\Delta_{n+1}\right\}=\left\{\Delta_{n}\right\}+\Delta t\left\{\dot{\Delta}_{n}\right\}+\Delta t^{2}(1 / 2-\zeta)\left\{\ddot{\Delta}_{n}\right\}+\Delta t^{2} \zeta\left\{\ddot{\Delta}_{n+1}\right\} \\
\left\{\dot{\Delta}_{n+1}\right\}=\left\{\dot{\Delta}_{n}\right\}+\Delta t(1-\gamma)\left\{\ddot{\Delta}_{n}\right\}+\Delta t \gamma\left\{\ddot{\Delta}_{n+1}\right\}
\end{gathered}
$$

Average-acceleration method for dynamic structures is one of the most common methods, which is unconditionally stable. In this method, the values are considered as $\gamma=0.5$ and $\zeta=0.25$ [19]. When the XFEM is used to simulate dynamic crack, network does not change over time $t_{n}$ to $t_{n+1}$. However, the new forms of functions are added to the model to simulate crack.

\section{Interaction integral}

Modes I and II of stress intensity factors cannot be calculated separately by calculating the integral J. In fact, interaction integral is the interaction of two independent and acceptable loading for cracks in structures, which is created in the elasticity conservative integral. In fact, integral interaction is an accurate formulation that enables us to calculate stress intensity factors in functional materials [19]. Implementation of interaction integral requires the use of auxiliary fields of displacement vector $u^{\text {aux }}$, strain $\varepsilon^{\text {aux }}$ and stress $\sigma^{\text {aux }}$ tensors. There are different choices for the auxiliary fields. In this study, the non-equilibrium formula to calculate interaction integral and stress intensity factors of functional materials will be used. 
Interaction integral formula can be written as [21]:

$$
I=-\frac{1}{R} \int\left[\operatorname{tr}(p \cdot \nabla q)+\left(\nabla \cdot p^{T}\right) q\right] r \mathrm{~d} A
$$

where $R$ is the radius of the crack tip, $A$ is the integration domain area (Fig. 2) and $I$ represents the volume integral. Thermal strain, inertia and inhomogeneity $\nabla \cdot p^{T}$ are zero in the absence of volume forces. The energy momentum tensor $p$ and vector $\vec{q}(r, z)$ are introduced according to Nahata and Moran [21] activities on the energy release rate for small cracks from equation (36).

$$
p_{l j}=w \delta_{l j}-\sigma_{i j} u_{i, l}
$$

$w$ is density of strain energy, $\sigma_{i j}$ and $u_{i, l}$ are components of stress tensor and displacement vector, respectively. Here $\vec{q}$ is considered as follows in cylindrical coordinates:

$$
\vec{q}=\left[\begin{array}{c}
q_{r} \\
q_{\theta} \\
q_{z}
\end{array}\right]=\left[\begin{array}{l}
q \\
0 \\
0
\end{array}\right]
$$

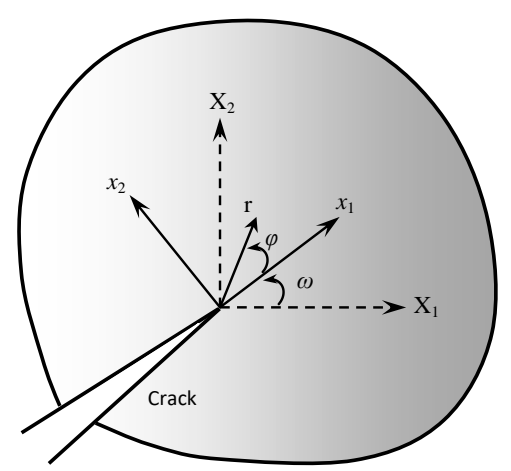

Fig. 2. Local $\left(x_{1}, x_{2}\right)$ and global $\left(\mathrm{X}_{1}, \mathrm{X}_{2}\right)$ coordinate systems [22]

In the most general case, interaction integral in cylindrical coordinates for a functional material can be stated as follows:

$$
\begin{aligned}
M I=\frac{1}{r_{c}} & \left\{\left(\sigma_{i r} u_{i, r}^{\mathrm{aux}}+\sigma_{i r}^{\mathrm{aux}} u_{i, r}-\sigma_{i j} \varepsilon_{i j}^{\mathrm{aux}}\right) q_{, r}+\left(\sigma_{\theta}^{\mathrm{aux}} \frac{u_{r}}{r}+\sigma_{\theta} \frac{u_{r}^{\mathrm{aux}}}{r}-\sigma_{i j} \varepsilon_{i j}^{\mathrm{aux}}\right) \frac{q}{r}\right. \\
& +\left(\sigma_{i z} u_{i, r}^{\mathrm{aux}}+\sigma_{i z}^{\mathrm{aux}} u_{i, r}\right) q_{, z}-\left[C_{i j k l, r} \varepsilon_{k l}^{m} \varepsilon_{i j}^{\mathrm{aux}}-\left(\rho \ddot{u}_{i}-B_{i}\right) u_{i, r}^{\mathrm{aux}}\right. \\
& -\sigma_{i j, j}^{\mathrm{aux}} u_{i, r}-\sigma_{k k}^{\mathrm{aux}}\left(\alpha_{, r} \theta+\alpha \theta_{, r}\right) \\
& \left.\left.+\frac{1}{r}\left(\sigma_{\theta}^{\mathrm{aux}} \frac{u_{r}}{r}+\sigma_{\theta} \frac{u_{r}^{\mathrm{aux}}}{r}-\sigma_{i r} u_{i, r}^{\mathrm{aux}}-\sigma_{i r}^{\mathrm{aux}} u_{i, r}\right)\right] q\right\} \mathrm{d} A
\end{aligned}
$$


The relation between the interaction integral and stress intensity factors for crack in plane strain case is as follows:

$$
M I_{l}=\frac{2}{E^{*}}\left(K_{I} K_{I}^{\mathrm{aux}}+K_{I I} K_{I I}^{\mathrm{aux}}\right)
$$

$E^{*}$ for axially symmetric problems is calculated identical to the plane strain problems.

$$
E^{*}=\frac{E_{\mathrm{tip}}}{\left(1-v_{\mathrm{tip}}^{2}\right)}
$$

In this relation, $E_{\text {tip }}$ and $v_{\text {tip }}$ are Young's modulus and Poisson's ratio at the crack tip, respectively. This relationship can be used for axial symmetrical problems. Put $K_{I I}^{\text {aux }}=0$ and $K_{I}^{\text {aux }}=1$ to calculate $K_{I}$ and consider $K_{I I}^{\text {aux }}=1$ and $K_{I}^{\text {aux }}=0$ to calculate $K_{I I}$ in the above equation.

\section{Modeling of functional materials}

In this study, it is assumed that material properties change occurs in the cylinder radial direction. In addition, it is assumed that the functional material is made of two components, matrix and the material added to it. In this study, the volume fraction of inclusion $V_{i}(r)$ follows the following exponential function.

$$
V_{i}(r)=\left(\frac{r-R}{w}\right)^{n}, \quad R \leqslant r \leqslant R+w
$$

$w$ is the cylinder thickness along the radius axis $r . R$ is the internal radius of the cylinder. Exponent $n$ determines the profile of the change of the material properties. Volume fraction of matrix is:

$$
V_{m}(r)=1-V_{i}(r)
$$

The density $\rho$, the specific heat capacity $c_{t}$, thermal expansion coefficient $\alpha$, and thermal conductivity coefficient $k$ are calculated using the rule of mixture in the functional material as equations from (44) to (47).

$$
\begin{gathered}
\rho=V_{i} \rho_{i}+V_{m} \rho_{m} \\
c_{t}=V_{i} c_{t i}+V_{m} c_{t m} \\
\alpha=V_{i} \alpha_{i}+V_{m} \alpha_{m} \\
k=V_{i} k_{i}+V_{m} k_{m}
\end{gathered}
$$


The shear rigidity modulus of matrix and inclusion is obtained as follows:

$$
\begin{gathered}
\mu_{i}=\frac{E_{i}}{2\left(1+v_{i}\right)} \\
\mu_{m}=\frac{E_{m}}{2\left(1+v_{m}\right)}
\end{gathered}
$$

where $E$ and $v$ are the Young's modulus and Poisson's ratio respectively. In addition, $i$ and $m$ specified inclusion and matrix respectively. $K$ is the bulk modulus and calculates as follows:

$$
\begin{gathered}
K_{i}=\frac{E_{i}}{3\left(1-2 v_{i}\right)} \\
K_{m}=\frac{E_{m}}{3\left(1-2 v_{m}\right)}
\end{gathered}
$$

To incorporate these relations into the XFE model, the value of each material property is calculated at each individual node based on the rule of mixture. Utilizing the generalized isoparametric graded finite elements, introduced by Kim and Paulino [23], material properties gradation is considered in an element. In the generalized isoparametric formulation, material properties at each Gaussian integration point can be interpolated from the nodal material properties of the element using the isoparametric shape functions which are the same for the spatial coordinates and displacements. Thus, material properties such as elastic modulus $(E)$, Poisson's ratio $(v)$, and mass density $(\rho)$ at Gauss points will be interpolated as follows [23]

$$
E=\sum_{i=1}^{4} N_{i} E_{i}, \quad v=\sum_{i=1}^{4} N_{i} v_{i}, \quad \rho=\sum_{i=1}^{4} N_{i} \rho_{1}
$$

For getting more information about precision of this formulation, one can refer to reference [23].

\section{Validation}

For the verification of the written code, four examples are discussed. The effect of heat and parameter $\mathrm{n}$ in functional material has been investigated in the first example. In the second example, the effect of rotation in the homogeneous material has been studied. In the third example, the effect of simple tension in a homogeneous cylinder with circumferential crack is investigated. In the fourth example, stress intensity factor for a cylinder under simple tension was examined and verified. 


\subsection{Thick-walled cylinder exposed to thermal shock transient}

Takabi [1] studied cylinder under mechanical and thermal load as numerical and analytical methods. This thick-walled cylinder was made of functional material with inner wall of the ceramic $\left(\mathrm{SiO}_{2}\right)$ and the outer wall of the metal $(\mathrm{Al})$. The boundary conditions were: $p_{\text {in }}=100 \mathrm{MPa}, p_{\text {out }}=10 \mathrm{MPa}, T_{\text {in }}=100{ }^{\circ} \mathrm{C}$ and $T_{\text {out }}=0{ }^{\circ} \mathrm{C}$. In this article as the first step, the cylinder in homogeneous case was studied at the period of 1000 seconds. The number of elements is $200 \times 50$. The mechanical properties of cylinder material are represented in Table 1.

Table 1.

Mechanical properties in the inner and outer wall [1]

\begin{tabular}{|c|c|c|c|c|c|c|}
\hline & $\begin{array}{c}\rho \\
\left(\mathrm{kg} / \mathrm{m}^{3}\right)\end{array}$ & $\begin{array}{c}E \\
(\mathrm{GPa})\end{array}$ & $\begin{array}{c}K \\
(\mathrm{~W} / \mathrm{m} \mathrm{K})\end{array}$ & $\begin{array}{c}\alpha \cdot 10^{-6} \\
(1 / \mathrm{K})\end{array}$ & $\begin{array}{c}C \\
(\mathrm{~J} / \mathrm{kg} \mathrm{K})\end{array}$ & $v$ \\
\hline$r_{1}=0.1 \mathrm{~m}$ & 5730 & 210 & 3.03 & 10.2 & 450 & 0.3 \\
$r_{2}=0.2 \mathrm{~m}$ & 7800 & 208 & 2 & 12 & 25.6 & 0.3 \\
\hline
\end{tabular}

Distribution of temperature and radial stress along the thickness of cylinder are compared with results of reference [1] in Fig. 3 and Fig. 4 respectively. Comparison of results shows that the obtained temperature distribution and radial stress is very close to the intended article.

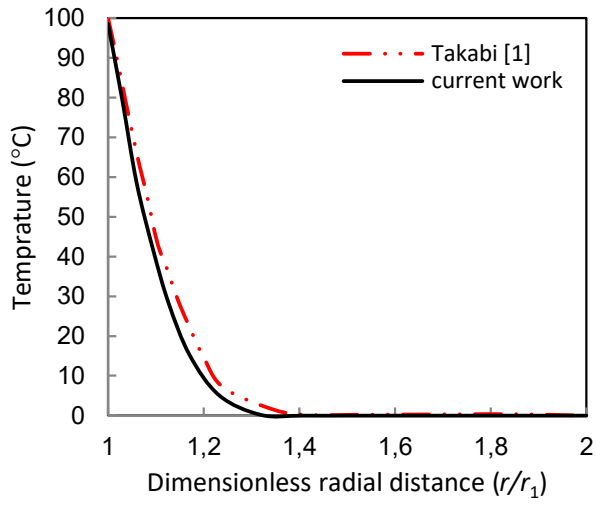

Fig. 3. Distribution of temperature along thickness of FG cylinder at the time of 1000 seconds

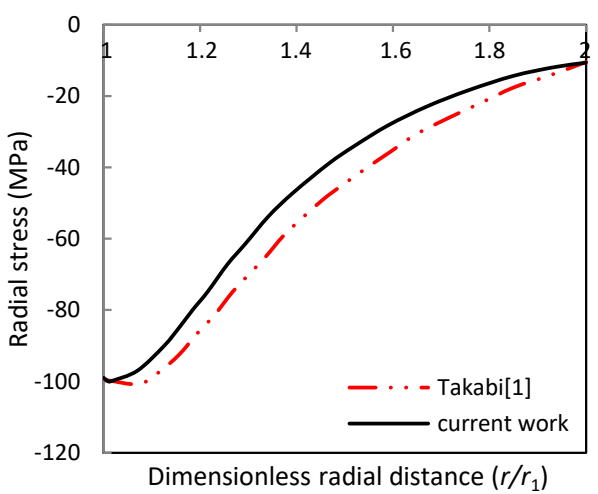

Fig. 4. Distribution of radial stress along thickness of FG cylinder at the time of 1000 seconds

\subsection{Thick-walled rotary FGM cylinder under dynamic loading}

Hearn [24] presented the formula for circumferential and radial stress analysis in homogeneous rotary hollow cylinder as follows:

$$
\sigma_{r}=\left(3+\frac{v}{1-v}\right)\left(\frac{\rho \omega^{2}}{8}\right)\left(R_{o}^{2}+R_{i}^{2}-\frac{R_{o}^{2} R_{i}^{2}}{r^{2}}-r^{2}\right)
$$




$$
\sigma_{H}=\left(\frac{\rho \omega^{2}}{8}\right)\left[\left(3+\frac{v}{1-v}\right)\left(R_{o}^{2}+R_{i}^{2}-\frac{R_{o}^{2} R_{i}^{2}}{r^{2}}\right)-\left(1+\frac{3 v}{1-v}\right) r^{2}\right]
$$

For this cylinder, the inner radius, wall thickness and the number of elements are selected as $r_{1}=0.4 \mathrm{~m}, t=0.2 \mathrm{~m}$ and $200 \times 50$, respectively. The angular velocity is considered $\omega=70 \mathrm{rad} / \mathrm{sec}$. The material properties of cylinder are in accordance with the Table 2. Distribution of temperature and hoop stress along the thickness of cylinder are illustrated in Fig. 5 and Fig. 6. In these figures, comparisons between the obtained results and analytical solution are done, which demonstrate the accuracy of the written code.

Table 2.

Isotropic cylinder properties

\begin{tabular}{|c|c|c|c|c|c|}
\hline $\begin{array}{c}\rho \\
\left(\mathrm{kg} / \mathrm{m}^{3}\right)\end{array}$ & $\begin{array}{c}E \\
(\mathrm{GPa})\end{array}$ & $\begin{array}{c}K \\
(\mathrm{~W} / \mathrm{m} \mathrm{K})\end{array}$ & $\begin{array}{c}\alpha \cdot 10^{-6} \\
(1 / \mathrm{K})\end{array}$ & $\begin{array}{c}C \\
(\mathrm{~J} / \mathrm{kg} \mathrm{K})\end{array}$ & $v$ \\
\hline 7850 & 200 & 2 & 12 & 25.6 & 0.3 \\
\hline
\end{tabular}

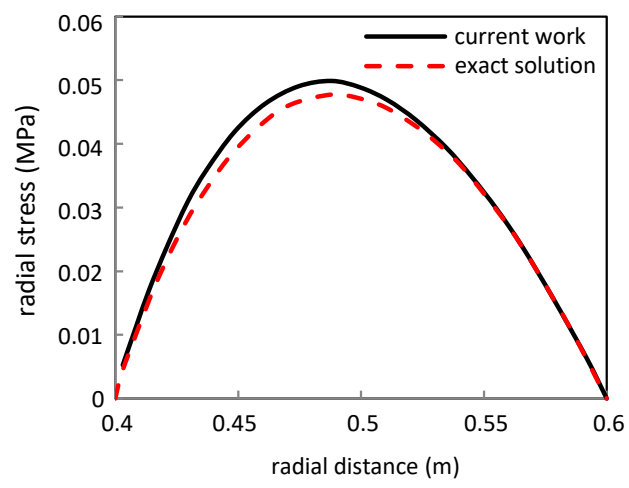

Fig. 5. Distribution of temperature along thickness of homogeneous cylinder at the time of 1000 seconds

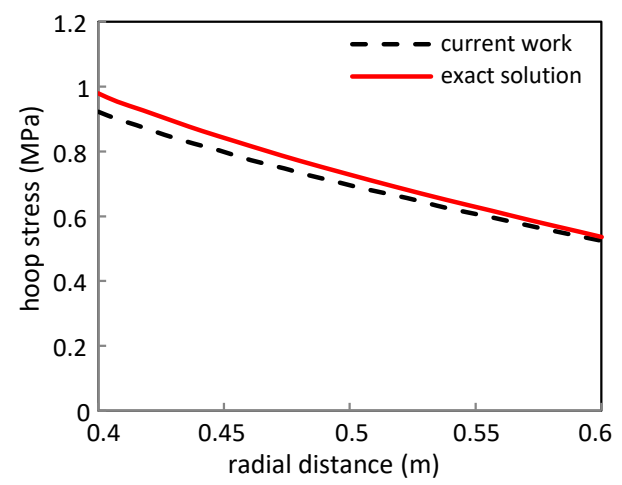

Fig. 6. Distribution of temperature along thickness of homogeneous cylinder at the time of 1000 seconds 


\subsection{Isotropic thick-walled cylinders with circumferential crack under axial tension}

Anderson [25] has provided the following formula for the stress intensity factor in a cylinder with circumferential crack under uniform tension.

$$
\begin{gathered}
K_{I}=\sigma_{t} \sqrt{\pi a} F_{t} \\
F_{t}=1.1+A\left[1.948\left(\frac{a}{t}\right)^{1.5}+0.3342\left(\frac{a}{t}\right)^{4.2}\right] \\
\text { if }: 5 \leqslant \frac{R_{i}}{t} \leqslant 10 \quad A=\left(0.125 \frac{R_{i}}{t}-0.25\right)^{0.25} \\
\text { if }: 10 \leqslant \frac{R_{i}}{t} \leqslant 20 \quad A=\left(0.4 \frac{R_{i}}{t}-3\right)^{0.25}
\end{gathered}
$$

where $K_{I}$ is the first mode stress intensity factor, $\sigma_{t}$ is the uniform axial stress, also $a, t$ and $R_{i}$ are circumferential crack depth, the cylinder thickness and internal radius of cylinder, respectively. In this example, $R_{i}=1 \mathrm{~m}, t=0.1 \mathrm{~m}, \sigma_{t}=1 \mathrm{MPa}$ and $a=0.05 \mathrm{~m}$ were assumed. A rectangular grid with $31 \times 61$ element is used. Isotropic material properties are presented in Table 2 . The stress intensity factor gained in exact solution of Anderson Equation and written program are $K_{I}=0.716$ $\mathrm{MPa} \cdot \mathrm{m}^{0.5}$ and $K_{I}=0.689 \mathrm{MPa} \cdot \mathrm{m}^{0.5}$, respectively. There is an about three percent difference between these results.

\subsection{Thin pipe with internal circumferential crack under uniform tension}

Grebner and Strathmeier [10] examined a pipe with through crack. In this study, a pipe with infinite length was put under axial loading. In this study, internal radius $R_{i}=50 \mathrm{~mm}$, pipe thickness $t=5 \mathrm{~mm}$, crack depth $a=2.5 \mathrm{~mm}$ Poisson's ratio $v=0.3$ and Young's modulus $E=2 \cdot 10^{5} \mathrm{MPa}$ were assumed. The axial stress on the pipe was $105 \mathrm{MPa}$. After inserting mentioned parameters and running the program, $K_{I}$ was found $K_{I}=557.41 \mathrm{Nmm}^{-1.5}$. Grebner [10] reported that this value was $520 \mathrm{Nmm}^{-1.5}$. As you can see, the amount of stress intensity factor in the first mode of failure in the written program is very close to the values reported.

\section{Numerical example}

In this section, we study the effects of pressure, temperature and angular velocity on the stress intensity factor in a FGM cylinder that includes a circumferential crack (Fig. 7). This cylinder was made of metal Ti-6Al-4V and ceramic $\mathrm{ZrO}_{2}$. The material properties have been chosen according to Table 3 . The inner radius is made of $\mathrm{ZrO}_{2}$ and the change of properties happens in $r$ direction. 


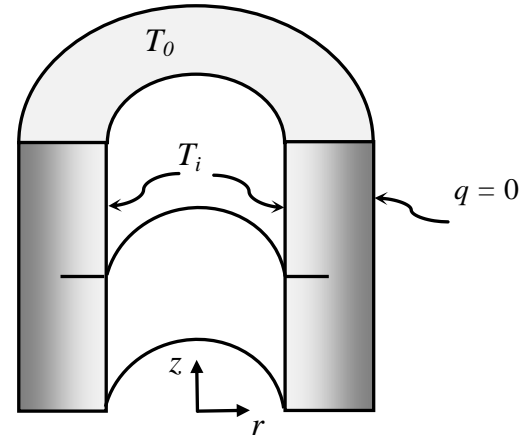

Fig. 7. Circumferential crack in a pressurized cylinder subject to transient thermal shock

Table 3.

The mechanical properties of titanium (metal) and zirconium (ceramic)

\begin{tabular}{|c|c|c|c|c|c|c|}
\hline & $\begin{array}{c}\rho \\
\left(\mathrm{kg} / \mathrm{m}^{3}\right)\end{array}$ & $\begin{array}{c}E \\
(\mathrm{GPa})\end{array}$ & $\begin{array}{c}K \\
(\mathrm{~W} / \mathrm{m} \mathrm{K})\end{array}$ & $\begin{array}{c}\alpha \cdot 10^{-6} \\
(1 / \mathrm{K})\end{array}$ & $\begin{array}{c}C \\
(\mathrm{~J} / \mathrm{kg} \mathrm{K})\end{array}$ & $v$ \\
\hline$R_{i}=0.1 \mathrm{~m}$ & 5600 & 117 & 2.03 & 7.11 & 516.6 & 0.33 \\
$R_{O}=0.2 \mathrm{~m}$ & 4410 & 66.2 & 18.1 & 10.3 & 808.3 & 0.32 \\
\hline
\end{tabular}

The change in the volume fraction of metal (Ti-6Al-4V) is calculated according to the formulas (42). Cylinder dimensions are: $R_{i}=0.1 \mathrm{~m}, R_{O}=0.2 \mathrm{~m}$ and cylinder height $H=0.2 \mathrm{~m}$. A grid with 12161 four-node rectangular elements is used to mesh the cylinder. To calculate the interaction integral, a square with four elements of length and width is considered.

\subsection{First example: Effect of material gradient parameter $(n)$ on the stress intensity factor}

In this example, we put the cylinder under cooling shock. The initial temperature is $400{ }^{\circ} \mathrm{C}$, at the moment $t=0 \mathrm{sec}$. A cooling shock of $200{ }^{\circ} \mathrm{C}$ is applied to the inner wall of cylinder. Crack depth is assumed $a=0.05 \mathrm{~m}$. Mechanical properties are shown in Table 3. Cylinder dimensions are $R_{i}=0.1 \mathrm{~m}, R_{O}=0.2 \mathrm{~m}$ and cylinder height is $H=0.2 \mathrm{~m}$.

The values of calculated stress intensity factors for various values of parameter $n$ are plotted as a curve in Fig. 8.

As shown, with the increasing $n$ for a given crack depth, the stress intensity factor is decreased in the first mode. The simulation stops before the reflected waves from the edges of the plate reach up to the crack tip. Therefore, the simulation time and applied time step will be $t=600 \mathrm{~s}$ and 10 seconds, respectively. Increasing parameter $n$ reduces the volume percentage of the metal and increases the ceramic phase within the cylinder. Thermal conductivity of ceramic is less than metal and this causes less heat flow to be transferred in the material. As a result, the stress 
intensity factor is reduced. Fig. 9 shows the stress intensity factor in terms of time for $n=0.6$ and crack depth $a=0.05 \mathrm{~m}$.

By applying the thermal load in the internal wall of cylinder so as to achieve a stress wave up to the crack tip, the stress intensity factor increases and after passing the stress wave, this factor drops.

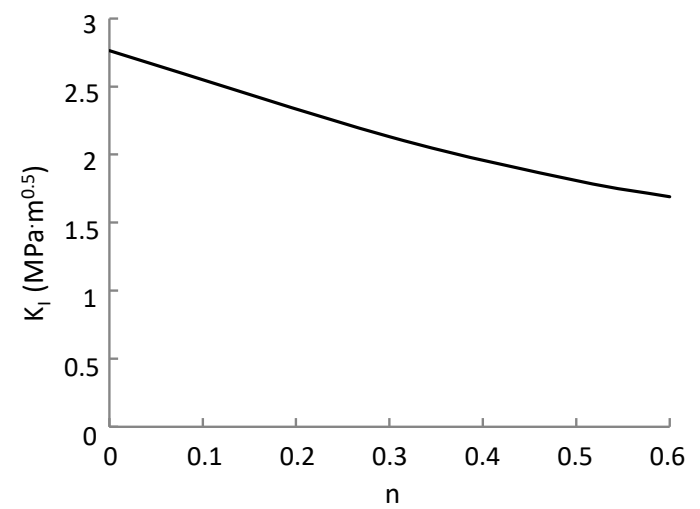

Fig. 8. Stress intensity factor versus various parameters $n$

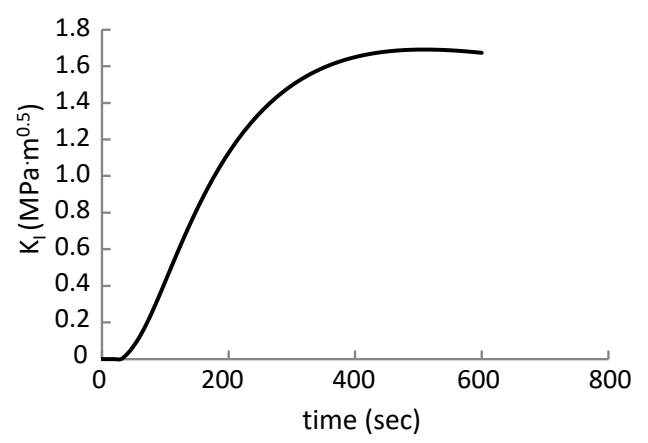

Fig. 9. The stress intensity factor in term of time for $a=0.05 \mathrm{~m}, n=0.6$

\subsection{Second example: Effect of the different cracks depth on the stress intensity factor}

In this example, we tend to study the first mode stress intensity factor for various crack depths ( $a=0.02, a=0.03, a=0.04, a=0.05$ and $a=0.06)$. The initial temperature is $400{ }^{\circ} \mathrm{C}$. At the moment $t=0$ a cooling shock of $200{ }^{\circ} \mathrm{C}$ is applied to the inner wall of cylinder. Changes in the stress intensity factor versus changes of parameter $n$ and the depth of crack are shown in Fig. 10. For any specific parameter $n$, increasing the depth of cracks reduces the stress intensity factor in the first mode.

With increasing the crack depth, the temperature in crack tip is reduced (because of the heat dissipation inside the material). This can reduce the stress intensity 


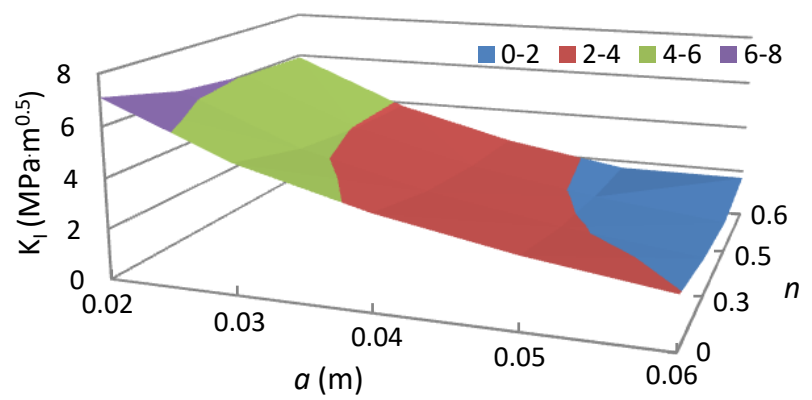

Fig. 10. 3D plot of maximum values of stress intensity factor in terms of crack depth and parameter $n$

factor. At any constant depth of crack, increasing parameter $n$ reduces the stress intensity factor. By increasing the parameter $n$, the percentage of the metal in the material reduces. The reducing metal phase leads to the thermal conductivity reduction in material and heat waves move slower in the cylinder and reduce the stress intensity factor.

\subsection{Third example: Effect of angular velocity on the stress intensity factor}

We put the cylinder under constant rotation $\omega=50 \mathrm{rad} / \mathrm{sec}$. The depth of circumferential crack is $a=0.05 \mathrm{~m}$. The analysis time was $300 \mathrm{sec}$. The temperature of the cylinder during the analysis was $200{ }^{\circ} \mathrm{C}$. The maximum values of stress intensity factor versus parameter $n$ curve is plotted in Fig. 11. Increasing parameter $n$ for a constant crack depth increases the stress intensity factor as shown in the Fig. 11.

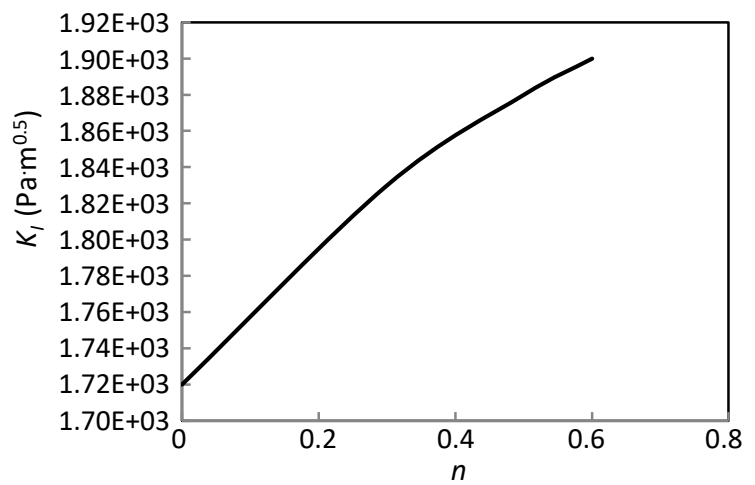

Fig. 11. The maximum amount of stress intensity factors for different parameter $n$

In this section we consider different crack depths. The cylinder is exposed to constant angular velocity $\omega=500 \mathrm{rad} / \mathrm{sec}$. Changes in the stress intensity factor versus changes of parameter $n$ and the depth of crack ars shown in Fig. 12. 


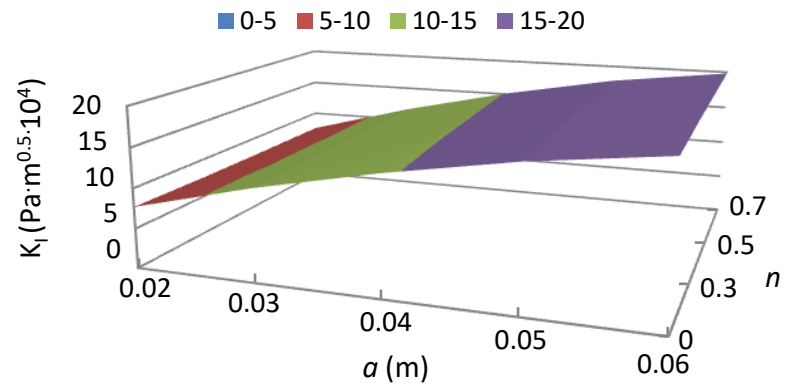

Fig. 12. The maximum amount of stress intensity factors for different $n$ and crack depth $a$

Obviously, increasing the depth of crack for a specific $n$ can increase the stress intensity factor. Now we consider the effects of different angular velocity on the maximum amount of stress intensity factor. The curve of maximum values of stress intensity factor versus angular velocity for $a=0.05$ and $n=0.6$ is plotted in Fig. 13.

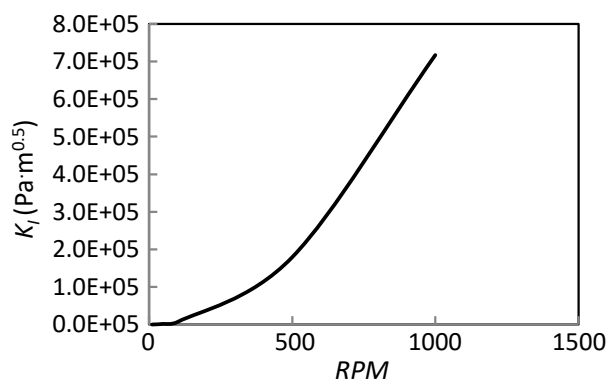

Fig. 13. The maximum amount of stress intensity factor changes vs. angular velocity

Temperature contours and radial and circumferential stresses with assumed $\omega=1000 \mathrm{rad} / \mathrm{sec}$ and $n=0.6$ at the time $t=500 \mathrm{sec}$ are shown in Figs. 14 and 15 , respectively.
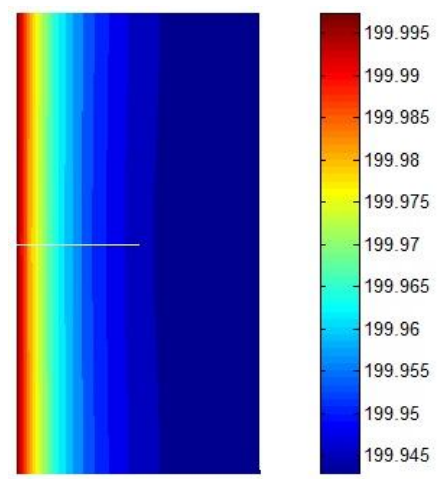

Fig. 14. Temperature contour $\left({ }^{\circ} \mathrm{C}\right)$ for $\omega=1000 \mathrm{rad} / \mathrm{sec}$ and $n=0.6$ 

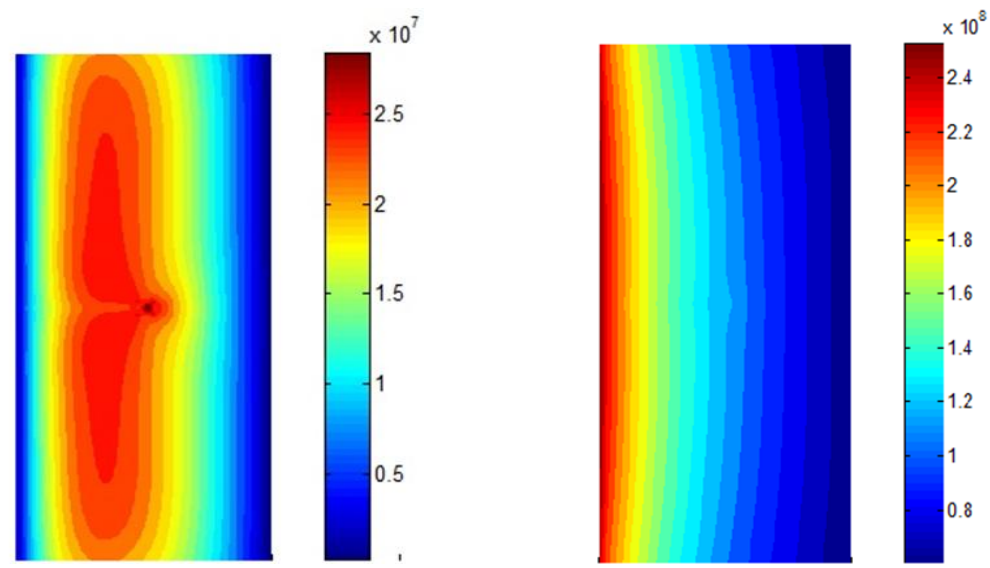

Fig. 15. a) Radial stress contour (Pa) b) Circumferential stress contour (Pa) for $\omega=1000 \mathrm{rad} / \mathrm{sec}$ and $n=0.6$

\subsection{Fourth example: Effect of combined loading; pressure, thermal shock and rotation}

In this study, the assumptions are: the internal radius of cylinder $r_{i}=0.1 \mathrm{~m}$, cylinder thickness $t=0.1 \mathrm{~m}$, angular velocity $\omega=10 \mathrm{rad} / \mathrm{sec}$, internal pressure $P_{\text {in }}=100 \mathrm{MPa}$ and initial temperature $T_{1}=273{ }^{\circ} \mathrm{C}$ and the temperature of internal wall of cylinder $T_{2}$ is $0{ }^{\circ} \mathrm{C}$. We extract the stress intensity factor for crack depth $a=0.05 \mathrm{~m}$ at $t=600 \mathrm{~s}$ for various parameters $n$. The simulation is done in a manner in which the reflected thermal waves from the edges do not reach up to the crack tip. The number of elements is $121 \times 61$. Also, the FGM is made of Ti-6Al-4V and $\mathrm{ZrO}_{2}$. Stress intensity factor changes in terms of time for various parameter $n$ are illustrated in Fig. 16.

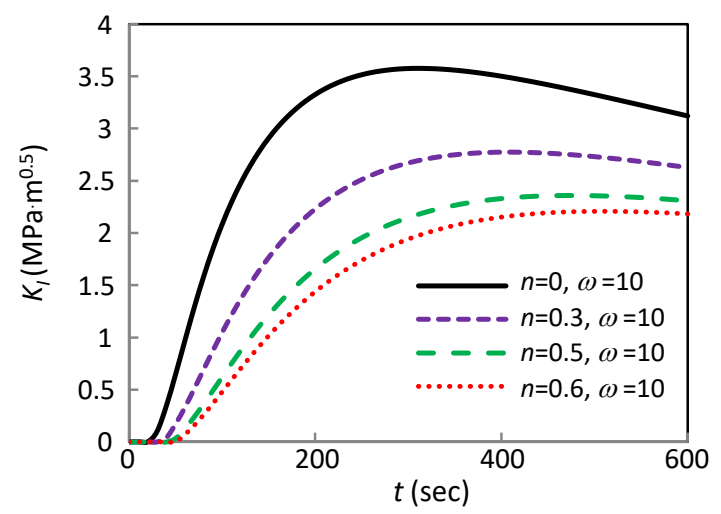

Fig. 16. Stress intensity factor vs. time curves for various parameters $n$ 
By increasing the parameter $n$ in a given angular velocity, the first mode stress intensity factor is reduced under internal pressure. Now we study the effect of different cracks depths on the stress intensity factor at a given parameter $n$. The stress intensity factor curves in terms of time for different crack depths are plotted in Fig. 17.

It is shown that increasing the crack depth, while the parameter $\mathrm{n}$ is constant, decreases the mode I stress intensity factor extremely.

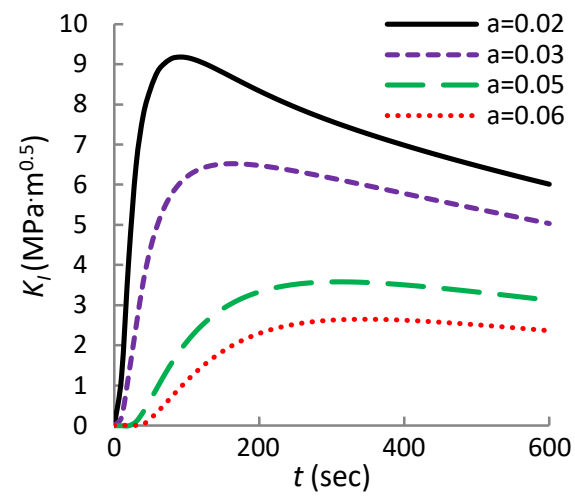

(a)

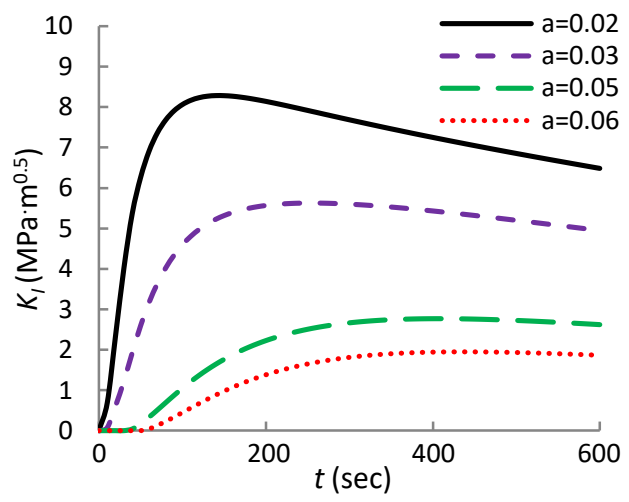

(b)

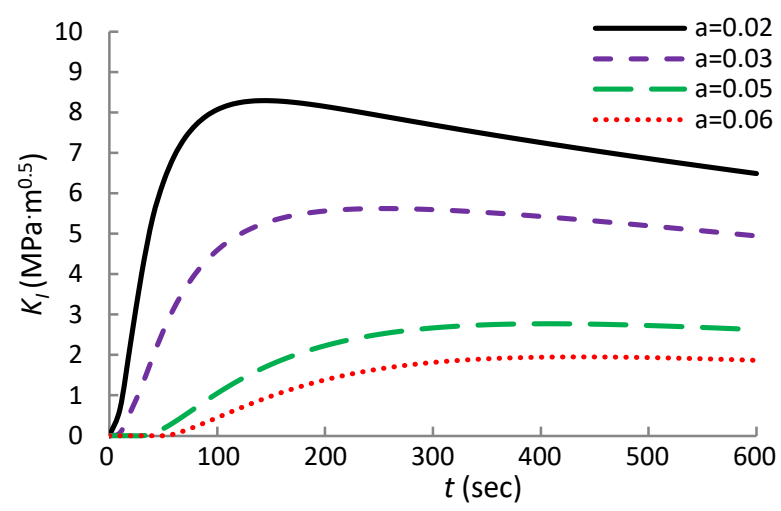

(c)

Fig. 17. Stress intensity factor changes in terms of time for different crack depths and a) $n=0$, b) $n=0.3$, c) $n=0.5$

In the following, the effect of angular velocity changes on the stress intensity factor is investigated. For this purpose a circumferential crack with depth $a=0.05$ $\mathrm{m}$ is considered. The parameter $n$ is 0.6 and analyses are done in compliance with all the initial presented assumptions.

The stress intensity factor vs. time curves are shown in Fig. 18. The results show that an increase in angular velocity increases the stress intensity factor. 


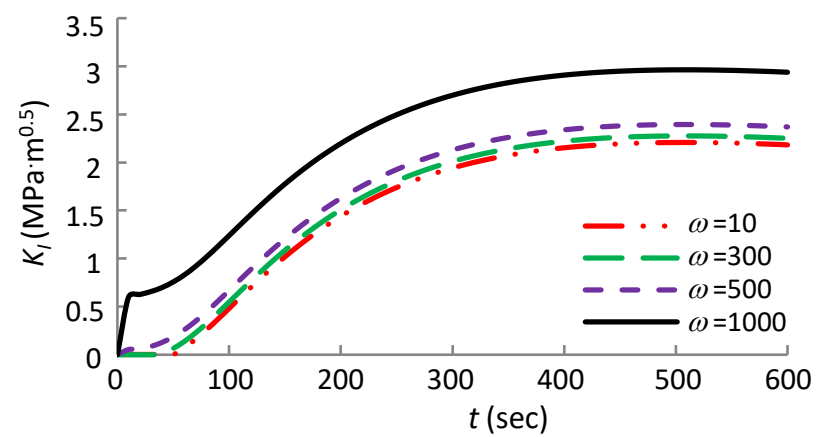

Fig. 18. Stress intensity factor variations in terms of time for $a=0.05 \mathrm{~m}, n=0.6$ and different angular velocity

\subsection{Fifth example: Effect of external pressure on stress intensity factor}

In this section, analyses are done with different external pressures, $P_{o}=10$, $100,200 \mathrm{MPa}$, internal pressure $P_{i}=10 \mathrm{MPa}$ and initial temperature $T_{1}=273$ ${ }^{\circ} \mathrm{C}$. The internal wall is subjected to a constant temperature cooling shock $T_{2}=0$ ${ }^{\circ} \mathrm{C}$. The default angular velocity is $\omega=10 \mathrm{rad} / \mathrm{sec}$. The stress intensity factor vs. time curves are shown in Fig. 19. The first mode stress intensity factor is reduced with increasing external pressure, as shown in Fig. 19. External pressure is partly responsible for opening the cracks.

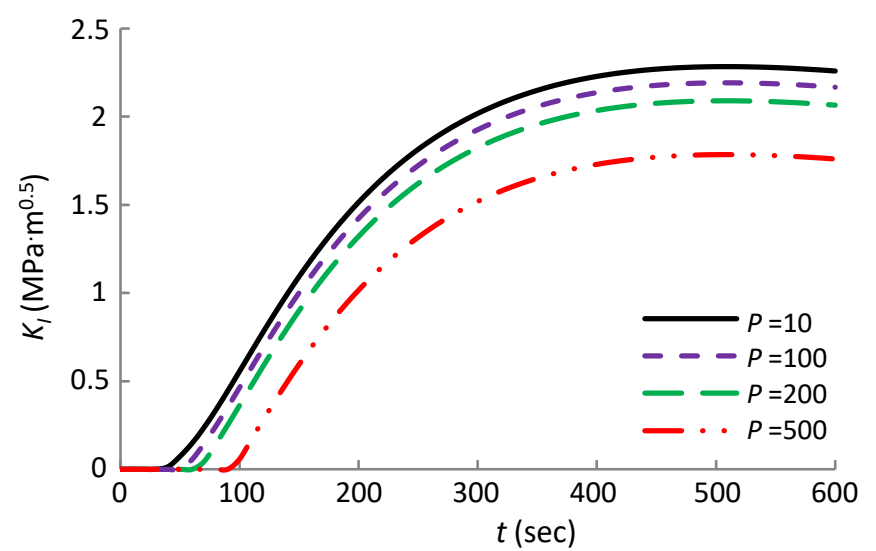

Fig. 19. Stress intensity factor vs. time curves for $n=0.6, a=0.05 \mathrm{~m}$ and various external pressures

\section{Conclusion}

In this paper, a circumferential crack in FGM cylinder under thermo-mechanical loading was studied. The classical coupled thermoelastic equations in cylindrical coordinates were solved by extended finite element and Newmark methods. FG 
material of cylinder was consisted of metal $\mathrm{ZrO}_{2}$ and ceramic Ti-6Al-4V. The inner radius was made of $\mathrm{ZrO}_{2}$ and the change of properties happened in the $r$ direction. The results of this study are as follows:

1. By increasing the parameter $n$, stress intensity factor decreases in the cylinder that has a circumferential crack with constant depth under axial tension.

2. When a cooling thermal shock is exerted on the inner wall, increasing the depth of crack with a given parameter $n$ reduces the stress intensity factor.

3. Under thermal shock, the stress intensity factor is reduced by increasing the parameter $n$.

4. At a given crack depth and a parameter $n$, increasing angular velocity increases the stress intensity factor in the cylinder.

5. At a given angular velocity and a parameter $n$, increasing the depth of crack increases the stress intensity factor.

6. In rotating cylinder with a specific crack depth, increasing the parameter $n$ increases the stress intensity factor.

7. At a given parameter $n$ and the constant crack depth, by increasing internal pressure one can decrease the stress intensity factor.

\section{A. Appendix}

Mass, damping and stiffness matrices, which are extracted from discretization of classical coupled thermoelastic equations.

$$
\begin{gathered}
\{\Delta\}=\left\{a_{h}^{u}, a_{h}^{w}, b_{h}^{u}, b_{h}^{w}, c_{h m}^{u}, c_{h m}^{w}, a_{h}^{T}, b_{h}^{T}, c_{1, m}^{w}\right\} \\
{[M]^{(e)}=\left[\begin{array}{cc}
{\left[M_{1}\right]} & {[0]_{48 \times 12}} \\
{[0]_{12 \times 48}} & {[0]_{12 \times 12}}
\end{array}\right]} \\
{\left[M_{1}\right]=\int_{V(e)} r \rho\left[B_{m}\right]^{T}\left[B_{m}\right] \mathrm{d} V=2 \pi \int_{V(e)} r^{2} \rho\left[B_{m}\right]^{T}\left[B_{m}\right] \mathrm{d} A} \\
{\left[C_{1}\right]=\int_{V(e)} T_{0} \beta\left[S_{t}\right]^{T}\left[S_{1}\right] \mathrm{d} V=2 \pi \int_{V(e)} T_{0} r \beta\left[S_{t}\right]^{T}\left[S_{1}\right] \mathrm{d} A} \\
{\left[C_{2}\right]=\int_{V(e)} r \rho c\left[S_{t}\right]^{T}\left[S_{1}\right] \mathrm{d} V=2 \pi \int_{V(e)} r^{2} \rho c\left[S_{t}\right]^{T}\left[S_{1}\right] \mathrm{d} A}
\end{gathered}
$$




$$
\begin{aligned}
& {[K]^{(e)}=\left[\begin{array}{cc}
{\left[K_{1}\right]} & {\left[K_{2}\right]} \\
{[0]_{12 \times 48}} & {\left[K_{3}\right]}
\end{array}\right]} \\
& {\left[K_{1}\right]=\int_{V(e)} r\left[B_{k}\right]^{T}[D]\left[B_{k}\right] \mathrm{d} V=2 \pi \int_{A(e)} r^{2}\left[B_{k}\right]^{T}[D]\left[B_{k}\right] \mathrm{d} A} \\
& {\left[K_{2}\right]=-\int_{V(e)} \beta\left[S_{1}\right]^{T}\left[S_{t}\right] \mathrm{d} V=-2 \pi \int_{A(e)} r \beta\left[S_{1}\right]^{T}\left[S_{t}\right] \mathrm{d} A} \\
& {\left[K_{3}\right]=\int_{V(e)} r k\left[S_{2}\right]^{T}\left[S_{2}\right] \mathrm{d} V=2 \pi \int_{A(e)} r^{2} k\left[S_{2}\right]^{T}\left[S_{2}\right] \mathrm{d} A} \\
& \{F\}^{(e)}=\left\{\begin{array}{c}
F_{r} \\
F_{z} \\
\int_{V(e)} r R S_{l} \mathrm{~d} V-\int_{A(e)}\left(r q_{r} n_{r}\right) S_{l} \mathrm{~d} A-\int_{A(e)}\left(r q_{z} n_{z}\right) S_{l} \mathrm{~d} A
\end{array}\right.
\end{aligned}
$$

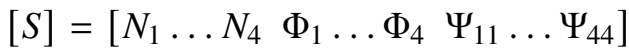

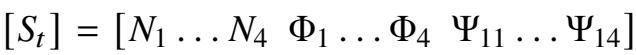

$$
\begin{aligned}
& {\left[S_{1}\right]=\left[r N_{1, r}+N_{1} \ldots r N_{4, r}+N_{4} r N_{1, z} \ldots r N_{4, z} r \varphi_{1, r}+\varphi_{1} \ldots r \varphi_{4, r}+\varphi_{4}\right.} \\
& \left.r \varphi_{1, z} \ldots r \varphi_{4, z} r \psi_{11, r}+\psi_{11} \ldots r \psi_{44, r}+\psi_{44} r \psi_{11, r} \ldots r \psi_{44, z}\right] \\
& {\left[S_{t}\right]=\left[r N_{1, r}+N_{1} \ldots r N_{4, r}+N_{4} r \varphi_{1, r}+\varphi_{1} \ldots r \varphi_{4, r}+\varphi_{4}\right.} \\
& r \psi_{11, r}+\psi_{11} \ldots r \psi_{44, r}+\psi_{44} \\
& \left.r N_{1, z} \ldots r N_{4, z} \quad r \varphi_{1, z} \ldots r \varphi_{4, z} \quad r \psi_{11, r} \ldots r \psi_{44, z}\right] \\
& {\left[S_{2}\right]=\left[N_{1, r} \ldots N_{4,4} \quad \varphi_{1, r} \ldots \varphi_{4, r} \quad \psi_{11, r} \ldots \psi_{44, r}\right.} \\
& \left.N_{1, z} \ldots N_{4, z} \quad \varphi_{1, z} \ldots \varphi_{4, z} \quad \psi_{11, z} \ldots \psi_{44, z}\right]
\end{aligned}
$$




\section{References}

[1] B. Takabi. Thermomechanical transient analysis of a thick-hollow FGM cylinder. Engineering Solid Mechanics, 4(1):25-32, 2016. doi: 10.5267/j.esm.2015.10.002.

[2] M. Shariati, M.M. Rokhi, and H. Rayegan. Investigation of stress intensity factor for internal cracks in FG cylinders under static and dynamic loading. Frattura ed Integrità Strutturale, (39):166-180, 2017. doi: 10.3221/IGF-ESIS.39.17.

[3] T. Meshii and K. Watanabe. Closed form stress intensity factor of an arbitrarily located inner-surface circumferential crack in an edge-restraint cylinder under linear radial temperature distribution. Engineering Fracture Mechanics, 60(5):519-527, 1998. doi: 10.1016/S00137944(98)00046-0.

[4] R. Seifi. Stress intensity factors for internal surface cracks in autofrettaged functionally graded thick cylinders using weight function method. Theoretical and Applied Fracture Mechanics, 75:113-123, 2015. doi: 10.1016/j.tafmec.2014.11.004.

[5] I. Eshraghi and N. Soltani. Stress intensity factor calculation for internal circumferential cracks in functionally graded cylinders using the weight function approach. Engineering Fracture Mechanics, 134:1-19, 2015. doi: 10.1016/j.engfracmech.2014.12.007.

[6] I.V. Varfolomeyev, M. Petersilge, and M. Busch. Stress intensity factors for internal circumferential cracks in thin-and thick-walled cylinders. Engineering Fracture Mechanics, 60(5):491-500, 1998. doi: 10.1016/S0013-7944(98)00045-9.

[7] Y.Z. Chen. Stress intensity factors in a finite length cylinder with a circumferential crack. International Journal of Pressure Vessels and Piping, 77(8):439-444, 2000. doi: 10.1016/S03080161(00)00047-8.

[8] I.S. Jones. Impulse response model of thermal striping for hollow cylindrical geometries. Theoretical and Applied Fracture Mechanics, 43(1):77-88, 2005. doi: 10.1016/j.tafmec.2004.12.004.

[9] A. Birinci, T.S. Ozsahin, and R. Erdol. Axisymmetric circumferential internal crack problem of a thick-walled cylinder with inner and outer claddings. European Journal of MechanicsA/Solids, 25(5):764-777, 2006. doi: 10.1016/j.euromechsol.2005.11.007.

[10] H. Grebner and U. Strathmeier. Investigation of different isoparametric axisymmetric crack tip elements applied to a complete circumferential surface crack in a pipe. Computers \& Structures, 21(6):1177-1180, 1985. doi: 10.1016/0045-7949(85)90172-5.

[11] V.-X. Tran and S. Geniaut. Development and industrial applications of X-FEM axisymmetric model for fracture mechanics. Engineering Fracture Mechanics, 82:135-157, 2012. doi: 10.1016/j.engfracmech.2011.12.002.

[12] T. Lewis and X. Wang. The T-stress solutions for through-wall circumferential cracks in cylinders subjected to general loading conditions. Engineering Fracture Mechanics, 75(10):32063225, 2008. doi: 10.1016/j.engfracmech.2007.12.001.

[13] R Ghajar and SM Nabavi. Closed-form thermal stress intensity factors for an internal circumferential crack in a thick-walled cylinder. Fatigue \& Fracture of Engineering Materials \& Structures, 33(8):504-512, 2010. doi: 10.1111/j.1460-2695.2010.01459.x.

[14] S. Navabi and M. Kamyab. Determination of transient thermal stress intensity factors in cylinders with circumferential crack. In 20th Annual International Conference on Mechanical Engineering, Shiraz, Iran, 2012.

[15] M. Shariati, H. Rayegan, and M.M. Rokhi. Calculation of stress intensity factors for FGM cylinder with central circular crack under static and dynamic loading. In 14th Conference of Iranian Aerospace Society, Tehran, Iran, 2015.

[16] M. Tehrani and T. Talebian. Analysis of FGM cylindrical vessels under thermo-mechanical loading. In Annual Conference on Mechanical Engineering, Tehran, Iran, 2008. 
[17] R.B. Hetnarski and M.R. Eslami. Thermal stresses: advanced theory and applications. Springer, 2009. doi: 10.1007/978-1-4020-9247-3.

[18] W.M. Lai, D. Rubin, and E. Krempl. Introduction to continuum mechanics. ButterworthHeinemann, 4th edition, 2009.

[19] M.M. Rokhi. Numerical analysis of crack propagation in a functionally graded layer under dynamic loading and thermal shock. Phd thesis, Faculty of Mechanics, Shahrood University of Technology, Iran, 2012.

[20] M.M. Rokhi and M. Shariati. Coupled thermoelasticity of a functionally graded cracked layer under thermomechanical shocks. Archives of Mechanics, 65(2):71-96, 2013.

[21] R. Nahta and B. Moran. Domain integrals for axisymmetric interface crack problems. International Journal of Solids and Structures, 30(15):2027-2040, 1993. doi: 10.1016/00207683(93)90049-D.

[22] M.M. Rokhi and M. Shariati. Implementation of the extended finite element method for coupled dynamic thermoelastic fracture of a functionally graded cracked layer. Journal of the Brazilian Society of Mechanical Sciences and Engineering, 35(2):69-81, 2013. doi: 10.1007/s40430013-0015-0.

[23] J.-H. Kim and G.H. Paulino. Isoparametric graded finite elements for nonhomogeneous isotropic and orthotropic materials. Journal of Applied Mechanics, 69(4):502-514, 2002. doi: 10.1115/1.1467094.

[24] E.J. Hearn. Mechanics of Materials. Butterworth-Heinemann, 1997.

[25] T.L. Anderson. Fracture Mechanics: Fundamentals and Applications. CRC Press, 2nd edition, 1994. 